Pacific Journal of Mathematics

TRIANGLE IDENTITIES AND SYMMETRIES OF A SUBSHIFT
OF FINITE TYPE

J 


\section{TRIANGLE IDENTITIES AND SYMMETRIES OF A SUBSHIFT OF FINITE TYPE}

\section{J. B. WAGONER}

We prove the group $\operatorname{Aut}\left(\sigma_{A}\right)$ of symmetries of a subshift of finite type is isomorphic to the fundamental group of the space $\operatorname{RS}(\mathscr{E})$ of strong shift equivalences built from the algebraic RS Triangle Identities for zero-one matrices which arise from triangles in the contractable simplicial complex of Markov partitions. Moreover, we show the higher homotopy groups of $\operatorname{RS}(\mathscr{E})$ are zero. $\operatorname{RS}(\mathscr{E})$ is therefore homotopy equivalent to the classifying space of $\operatorname{Aut}\left(\sigma_{A}\right)$.

1. Introduction and statement of results. First we briefly review Williams' strong shift equivalence criterion for conjugacy of subshifts of finite type. See $[3,4,8]$. Let $A: \mathscr{S} \times \mathscr{S} \rightarrow\{0,1\}$ and $B: \mathscr{T} \times \mathscr{T} \rightarrow$ $\{0,1\}$ be zero-one matrices on the finite state spaces $\mathscr{S}$ and $\mathscr{T}$. An elementary strong shift equivalence

$$
(R, S): A \rightarrow B
$$

is a pair of zero-one matrices $R: \mathscr{S} \times \mathscr{T} \rightarrow\{0,1\}$ and $S: \mathscr{T} \times \mathscr{S} \rightarrow$ $\{0,1\}$ satisfying

$$
R S=A \quad \text { and } \quad S R=B .
$$

Let $\left(X_{A}, \sigma_{A}\right)$ and $\left(X_{B}, \sigma_{B}\right)$ be the subshifts of finite type (SFT) constructed from $A$ and $B$ respectively. The strong shift equivalence $(R, S)$ gives rise to an elementary symbolic conjugacy

$$
c(R, S): X_{A} \rightarrow X_{B}
$$

defined as follows: Let $x=\left\{x_{n}\right\}$ be in $X_{A}$. Then $y=c(R, S)(x)$ is the unique point $y=\left\{y_{n}\right\}$ in $X_{B}$ such that $1=A\left(x_{n}, x_{n+1}\right)=$ $R\left(x_{n}, y_{n}\right) S\left(y_{n}, x_{n+1}\right)$ for all $n$. Similarly, one has

$$
c(S, R): X_{B} \rightarrow X_{A}
$$

and it is easy to verify the identities

$$
c(S, R) c(R, S)=\sigma_{A} \quad \text { and } \quad c(R, S) c(S, R)=\sigma_{B}
$$

which show that $c(R, S)$ and $c(S, R)$ are conjugacies. More generally, let $\mathscr{E}$ denote the set of zero-one matrices on finite state spaces. We shall assume that any matrix in $\mathscr{E}$ has at least one non-zero entry 
in each row and in each column. Williams defined $A$ and $B$ to be strong shift equivalent in $\mathscr{E}$ provided there is a chain of elementary strong shift equivalences from $A$ to $B$ through intermediate matrices in $\mathscr{E}$. The composition of the corresponding elementary conjugacies gives a conjugacy from $\left(X_{A}, \sigma_{A}\right)$ to $\left(X_{B}, \sigma_{B}\right)$. Williams' proof of the converse that conjugacy implies strong shift equivalence brings in the set $P_{A}$ of Markov partitions for $\sigma_{A}$ on $X_{A}$. In [8] $P_{A}$ is given the structure of a locally finite simplicial complex in which each Markov partition is a vertex, and the key step in Williams' argument really amounts to showing that $P_{A}$ is connected. In fact, $P_{A}$ turns out to be contractable [8], and in this paper we make use of the fact that it is simply connected to prove (1.5) and contractability to prove (1.13) below.

The definition of Markov partition used in [8] for $P_{A}$ is the one in [5]. A similar theory goes through using the Markov partitions by rectangles as presented, say, in [4, p. 100]. Let $U=\left\{U_{i}\right\}$ be in $P_{A}$ and let $M=M(U)=\{M(i, j)\}$ be the zero-one matrix where

$$
M(i, j)=1 \text { iff } U_{i} \cap \sigma_{A}^{-1}\left(U_{j}\right) \neq \varnothing .
$$

For example, $A=M\left(U^{A}\right)$ where $U^{A}=\left\{U_{i}^{A}\right\}$ is the "standard" Markov partition with $U_{i}^{A}$ equal to the cylinder set of those $x=\left\{x_{n}\right\}$ such that $x_{0}=i$. Let $V=\left\{V_{k}\right\}$ also be in $P_{A}$. Define zero-one matrices $R=R(U, V)=\{R(i, k)\}$ and $S(V, U)=\{S(k, i)\}$ by the formulas

$$
\begin{gathered}
R(i, k)=1 \text { if and only if } U_{i} \cap V_{k} \neq \varnothing, \\
S(k, i)=1 \text { if and only if } V_{k} \cap \sigma_{A}^{-1}\left(U_{i}\right) \neq \varnothing .
\end{gathered}
$$

Write $U<V$ to mean that $V$ refines $U$. As in [8] we write $U \rightarrow V$ provided $U<V<U \cap \sigma_{A}^{-1}(U)$, and we write $U \rightarrow V$ provided $U<V<\sigma_{A}(U) \cap U$. Finally, we write $U \rightarrow V$ iff $U \rightarrow U \cap V \leftarrow V$. An (ordered) 1-simplex of $P_{A}$ is a pair $[U, V]$ where $U \neq V$ and $U \rightarrow V$. Let $P=M(U), Q=M(V)$, and $R$ and $S$ be as above. It was shown in [8] that

$$
P=R S \text { and } Q=S R
$$

whenever $U \rightarrow V$; that is, $(R, S): P \rightarrow Q$. In particular, connectivity of $P_{A}$ implies the transition matrices $M(U)$ and $M(V)$ of any two Markov partitions $U$ and $V$ in $P_{A}$ are strong shift equivalent in $\mathscr{E}$. To finish off the outline of Williams' proof, let $\alpha:\left(X_{A}, \sigma_{A}\right) \rightarrow$ $\left(X_{B}, \sigma_{B}\right)$ be a topological conjugacy. Let $U=\left\{U_{i}\right\}$ be in $P_{A}$ where 
$i$ runs through an indexing set $I$. Then $\alpha(U)=\left\{\alpha\left(U_{i}\right)\right\}$ is in $P_{B}$. In fact, let $V=\left\{V_{k}\right\}$ be in $P_{B}$ where $k$ is in the index set $K$, and suppose we have $V=\alpha(U)$; that is, each $V_{k}=\alpha\left(U_{i}\right)$ for exactly one $U_{i}$. Then $\alpha$ gives a bijection $\alpha: I \rightarrow K$. Throughout this paper we will follow the convention that $\alpha$ also denotes the $K \times I$ permutation matrix with $\alpha(k, i)=1$ iff $\alpha(i)=k$. Let $P=M(U)$ and $Q=M(V)$. Since $U_{i} \cap \sigma_{A}^{-1}\left(U_{j}\right) \neq \varnothing$ iff $\alpha\left(U_{i}\right) \cap \sigma_{A}^{-1}\left(\alpha\left(U_{j}\right)\right) \neq \varnothing$, we have $Q=\alpha P \alpha^{-1}$. Therefore

$$
\left(\alpha^{-1}, \alpha P\right): P \rightarrow Q
$$

Hence $A$ is strong shift equivalent to $B$.

Let $\operatorname{Aut}\left(\sigma_{A}\right)$ be the group of symmetries of $\left(X_{A}, \sigma_{A}\right)$. By definition, this is the group of homeomorphisms of $X_{A}$ which commute with $\sigma_{A}$. It is discrete in the topology of uniform convergence, because $\sigma_{A}$ is expansive. See $[\mathbf{1}, \mathbf{2}, \mathbf{8}]$ for some recent information about this group. The preceding discussion suggests that elements of $\operatorname{Aut}\left(\sigma_{A}\right)$ can be described as products of various elementary conjugacies $c(R, S)$ modulo certain relations. This turns out to be the case, and a very natural set of relations which do work come from triangles in $P_{A}$.

By definition a triangle in $P_{A}$ is an ordered triple $[U, V, W]$ of Markov partitions such that $U \rightarrow V, V \rightarrow W$, and $U \rightarrow W$. Let

$$
\begin{array}{ll}
M=M(U), & P=M(V), \quad Q=M(W), \\
R_{1}=R(U, V), & S_{1}=S(V, U), \\
R_{2}=R(V, W), & S_{2}=S(W, V), \\
R_{3}=R(U, W), & S_{3}=S(W, U) .
\end{array}
$$

In $\S 2$ we will verify the $R S$ Triangle Identities:

$$
R_{1} R_{2}=R_{3}, \quad R_{2} S_{3}=S_{1}, \quad S_{3} R_{1}=S_{2} .
$$

Upon either multiplying the second equation on the left by $S_{2}$ or by multiplying the third equation on the right by $S_{1}$, we derive the $S S$ Triangle Identities found in [8]:

$$
\begin{aligned}
R_{1} R_{2} & =R_{3}, \\
S_{2} S_{1} & =Q S_{3}=S_{3} M .
\end{aligned}
$$

DEFINITION 1.4 The space $R S(\mathscr{E})$ of strong shift equivalence in $\mathscr{E}$ based on the RS Triangle Identities is the geometric realization of the simplicial set where an $n$-simplex consists of the following data:

(a) an $(n+1)$-tuple $\left\langle A_{0}, \ldots, A_{n}\right\rangle$ of square matrices in $\mathscr{E}$ and

(b) for each $i<j$ a strong shift equivalence $\left(R_{i j}, S_{j i}\right): A_{i} \rightarrow A_{j}$ in 
$\mathscr{E}$ such that the RS Triangle Identities hold for $i<j<k$; that is,

$$
R_{i j} R_{j k}=R_{i k}, \quad R_{j k} S_{k i}=S_{j i}, \quad S_{k i} R_{i j}=S_{k j} .
$$

The face operators are the usual forgetful ones and the degeneracies insert the strong shift equivalence $\left(1, A_{i}\right)$ from $A_{i}$ to itself. See [6] or [7] for background on simplicial sets and $\mathrm{CW}$ complexes. It is immediate from the definition that the set of components $\pi_{0}(R S(\mathscr{E}))$ is exactly the set of strong shift equivalences in $\mathscr{E}$.

THEOREM 1.5. There is an isomorphism

$$
\Phi_{A}: \operatorname{Aut}\left(\sigma_{A}\right) \rightarrow \pi_{1}(\operatorname{RS}(\mathscr{E}), A) .
$$

The explicit formula for $\Phi_{A}$ is given in (3.5) and (3.8) below.

For any strong shift equivalence $(R, S): P \rightarrow Q$, let $\gamma(R, S)$ denote the corresponding homotopy class of paths from $P$ to $Q$ in $\mathrm{RS}(\mathscr{E})$. Elementary arguments in algebraic topology show that elements $\gamma$ in $\pi_{1}(\mathrm{RS}(\mathscr{E}), A)$ can be represented as products

$$
\gamma=\prod_{i=1}^{n} \gamma\left(R_{i}, S_{i}\right)^{\varepsilon_{i}}
$$

where $\varepsilon_{i}= \pm 1$. The defining relations are

$$
\gamma(R, S) \gamma(R, S)^{-1}=1
$$

and

$$
\gamma\left(R_{1}, S_{1}\right) \gamma\left(R_{2}, S_{2}\right)=\gamma\left(R_{3}, S_{3}\right)
$$

whenever the RS Triangle Identites hold; that is,

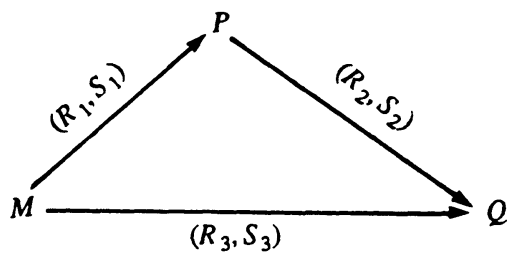

is a triangle in $\operatorname{RS}(\mathscr{E})$. Given this presentation of $\pi_{1}(\operatorname{RS}(\mathscr{E}), A)$, the inverse

$$
\Theta_{A}: \pi_{1}(\operatorname{RS}(\mathscr{E}), A) \rightarrow \operatorname{Aut}\left(\sigma_{A}\right)
$$


of $\Phi_{A}$ is easy to describe: namely, $\Theta_{A}$ takes the product (1.6) to

$$
\Theta_{A}(\gamma)=\prod_{i=1}^{n} c\left(R_{i}, S_{i}\right)^{-\varepsilon_{i}}
$$

where composition is read from right to left. That this is well defined follows immediately from

Lemma 1.9. Suppose $\left(R_{1}, S_{1}\right): M \rightarrow P,\left(R_{2}, S_{2}\right): P \rightarrow Q$, and $\left(R_{3}, S_{3}\right): M \rightarrow Q$. Assume that $R_{1} R_{2}=R_{3}$. Then

$$
c\left(R_{2}, S_{2}\right) c\left(R_{1}, S_{1}\right)=c\left(R_{3}, S_{3}\right)
$$

if and only if $R_{2} S_{3}=S_{1}$ and $S_{3} R_{1}=S_{2}$.

The first equation of the RS Triangle Identities is very reasonable. This lemma shows that the last equations, which were harder to guess, are exactly what is needed to make composition of elementary conjugacies behave well around a triangle.

There is also the space $\mathrm{SS}(\mathscr{E})$ of strong shift equivalence in $\mathscr{E}$ based on the SS Triangle Identities with the same face and degeneracy operators as used for $\mathrm{RS}(\mathscr{E})$. Since the RS Identities imply the SS identities, there is a natural continuous map

$$
\mathrm{RS}(\mathscr{E}) \rightarrow \mathrm{SS}(\mathscr{E})
$$

The set of components $\pi_{0}(\mathrm{SS}(\mathscr{E}))$ is also the set of strong shift equivalence in $\mathscr{E}$ and the induced map $\pi_{0}(\operatorname{RS}(\mathscr{E})) \rightarrow \pi_{0}(\operatorname{SS}(\mathscr{E}))$ is a bijection. The commutative diagram (4.30) of [8] expands to

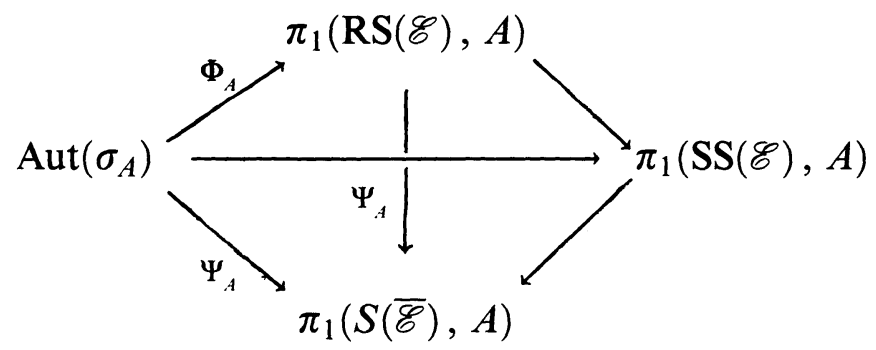

where $S(\overline{\mathscr{E}})$ is the space of shift equivalences of non-negative integral matrices. It was shown in [8] there is an isomorphism between $\pi_{1}(S(\overline{\mathscr{E}}), A)$ and the $\operatorname{group} \operatorname{Aut}\left(s_{A}\right)=\operatorname{Aut}\left(G(A), G(A)_{+}, s_{A}\right)$ of order preserving automorphisms of the dimension group $G(A)$ which commute with the automorphism $s_{A}$ induced on $G(A)$ by $A$. The Finite 
Order Generation Conjecture (FOG) can therefore be reformulated to state

The kernel of

$$
\delta_{A}: \pi_{1}(\mathrm{RS}(\mathscr{E}), A) \rightarrow \pi_{1}(S(\overline{\mathscr{E}}), A)
$$

is generated by elements of finite order.

In [9] we use the spaces $\operatorname{RS}(\mathscr{E})$ and $S(\overline{\mathscr{E}})$ together with methods of this paper to prove the following eventual version of this conjecture:

Eventual fog 1.12. Let $\alpha$ be in $\operatorname{ker}\left(\delta_{A}\right)$. There is an integer $k_{0} \geq 1$ such that if $k \geq k_{0}$, then $\alpha$ is a product of homeomorphisms of $X_{A}$ of finite order which commute with $\sigma_{A}^{k}$.

In the proof of (1.12) given in [9], the integer $k_{0}$ seems to depend on $\alpha$ and the product expressions are possibly different for different $k$. Of course, FOG conjectures that we can take $k_{0}=1$, in which case the product expression for $k=1$ works for all $k$. M. Boyle has shown $\alpha$ in (1.12) is a product of just two finite order elements commuting with $\sigma_{A}^{k}$ for large $k$, although in practice one must take $k$ larger than $k_{0}$ to do this.

In $\S 4$ we prove

TheOREM 1.13. $\pi_{n}(\operatorname{RS}(\mathscr{E}), A)=0$ for $n \geq 2$.

For simplicity, we assume throughout this paper that all the matrices in $\mathscr{E}$ are finite. However, (1.5) and (1.13) generalize without change when $\mathscr{E}$ consists of infinite matrices in one of the three classes considered in (4.1) of [8]. There is also a stochastic version for symmetries of $\left(X_{A}, \sigma_{A}\right)$ which preserve a $\sigma_{A}$-invariant Markov measure.

2. Proof of the RS triangle identities. Consider the triangle

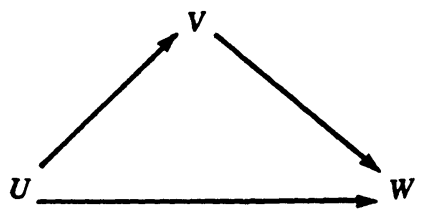

in $P_{A}$ with the corresponding matrices (1.1). The equation $R_{1} R_{2}=$ $R_{3}$ was verified in (3.3) of [8]. So it remains to prove the last two 
equations in (1.2). As in $\S 3$ of [8] this subdivides into 9 triangles

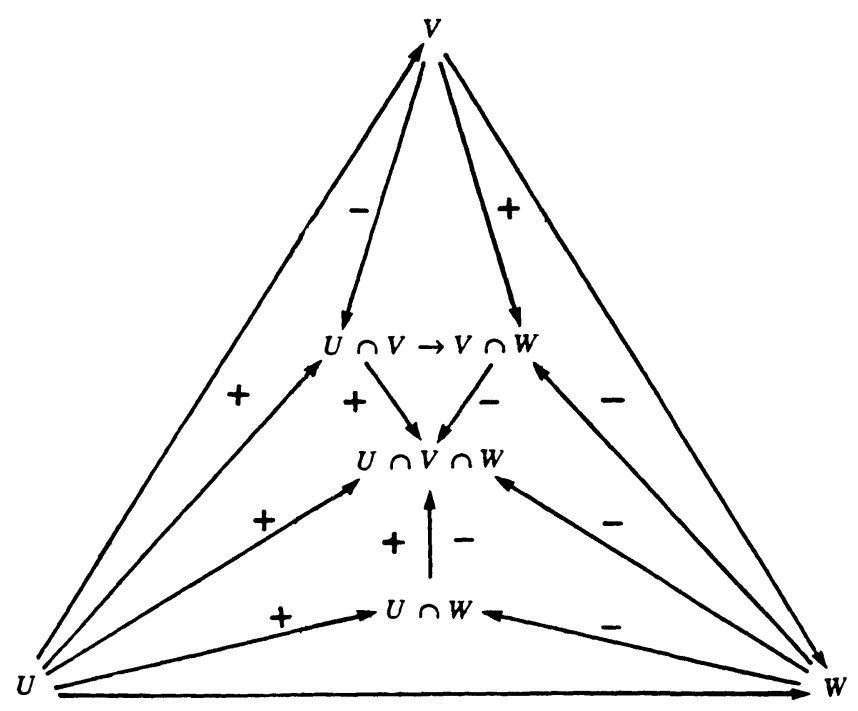

As in (3.9) of [8] we have $U \cap W=U \cap V \cap W$ and the diagram

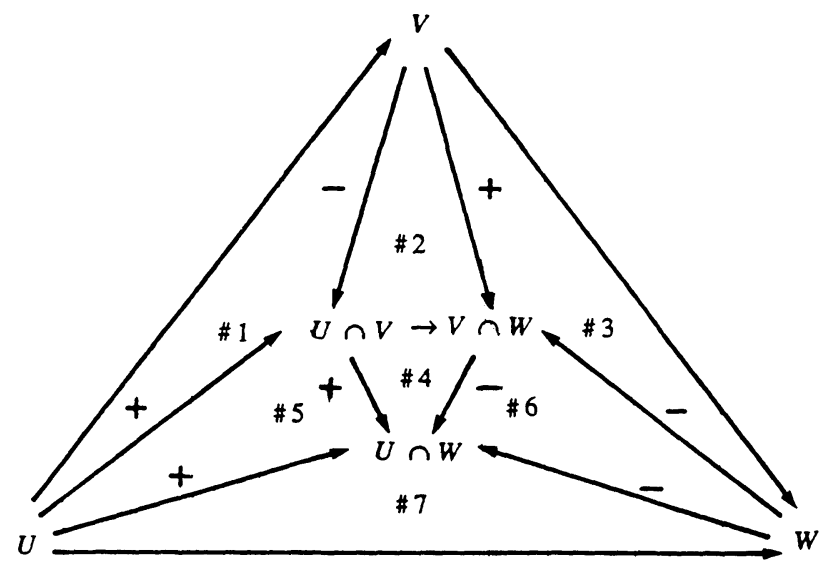

The first step is to verify the RS Triangle Identities for each triangle in (2.3). In all that follows, "LHS" is the left hand side of an equation and "RHS" is the right hand side. Let $\sigma=\sigma_{A}$.

Triangles \#1, \#3, \#4, \#7:

These are all of the form

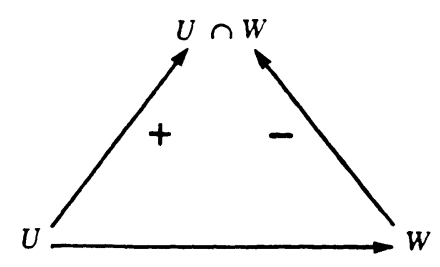


(I) $S_{1}=R_{2} S_{3}$.

LHS: $S_{1}((i, p), j)=1$ iff $U_{i} \cap W_{p} \cap \sigma^{-1}\left(U_{j}\right) \neq 0$.

RHS: The term $R_{2}((i, p), q) S_{3}(q, j)=1$ iff $U_{i} \cap W_{p} \cap W_{q} \neq \varnothing$ and $W_{q} \cap \sigma^{-1}\left(U_{j}\right) \neq \varnothing$.

In particular $q=p$; so there is at most one non-zero term and the RHS is a zero-one matrix.

Write

$$
U_{i}=\bigcup \sigma\left(W_{a}\right) \cap W_{b}, \quad U_{j}=\bigcup \sigma\left(W_{c}\right) \cap W_{d} .
$$

Assume RHS $\neq 0$. Then $U_{i} \cap W_{p} \neq \varnothing$ implies some $b=p$ and $W_{q} \cap \sigma^{-1}\left(U_{j}\right) \neq \varnothing$ implies some $c=q=p$. Therefore,

$$
U_{i} \cap \sigma^{-1}\left(U_{j}\right) \supset \sigma\left(W_{a}\right) \cap W_{p} \cap \sigma^{-1}\left(W_{d}\right) \neq \varnothing
$$

for some $a$ and some $d$. This shows $U_{i} \cap W_{p} \cap \sigma^{-1}\left(U_{j}\right) \neq 0$ implying LHS $\neq 0$. Conversely, suppose that LHS $\neq 0$. Then $U_{i} \cap W_{p} \cap$ $\sigma^{-1}\left(U_{j}\right) \neq 0$. Let $q=p$. Then $U_{i} \cap W_{p} \cap W_{q} \neq \varnothing$ and $W_{q} \cap \sigma^{-1}\left(U_{j}\right) \neq$ $\varnothing$ implying RHS $\neq 0$.

(II) $S_{2}=S_{3} R_{1}$.

LHS: $S_{2}(p,(j, q))=1$ iff $W_{p} \cap \sigma^{-1}\left(U_{j}\right) \cap \sigma^{-1}\left(W_{q}\right) \neq \varnothing$.

RHS: Consider a single term $S_{3}(p, i) R_{1}(i,(j, q))$. This is equal to 1 iff $W_{p} \cap \sigma^{-1}\left(U_{i}\right) \neq \varnothing$ and $U_{i} \cap U_{j} \cap W_{q} \neq \varnothing$; which implies $i=j$ and $U_{i} \supset U_{j} \cap W_{q}$.

Thus there is at most one $i$ giving a non-zero term on the RHS, and so $S_{3} R_{1}$ is a zero-one matrix.

Now write

$$
W_{p}=\bigcup U_{a} \cap \sigma^{-1}\left(U_{b}\right), \quad W_{q}=\bigcup U_{e} \cap \sigma^{-1}\left(U_{f}\right) .
$$

Assume RHS $\neq 0$. Then $i=j$ and

$$
W_{p} \cap \sigma^{-1}\left(U_{i}\right)=\bigcup U_{a} \cap \sigma^{-1}\left(U_{b}\right) \cap \sigma^{-1}\left(U_{j}\right)
$$

implying some $b=j$. Similarly,

$$
U_{j} \cap W_{q}=\bigcup U_{j} \cap U_{e} \cap \sigma^{-1}\left(U_{f}\right)
$$

implying some $e=j$. Therefore,

$$
W_{p} \cap \sigma^{-1}\left(U_{j}\right) \cap \sigma^{-1}\left(W_{q}\right) \supset U_{a} \cap \sigma^{-1}\left(U_{j}\right) \cap \sigma^{-2}\left(U_{f}\right) \neq \varnothing
$$

for some $a$ and some $f$. Hence LHS $\neq 0$. Conversely, suppose LHS $\neq 0$. Then $W_{p} \cap \sigma^{-1}\left(U_{j}\right) \neq \varnothing$ and $U_{j} \cap U_{j} \cap W_{q} \neq \varnothing$ implying RHS $\neq 0$. 
Triangles \#5, \#6:

These are of the form

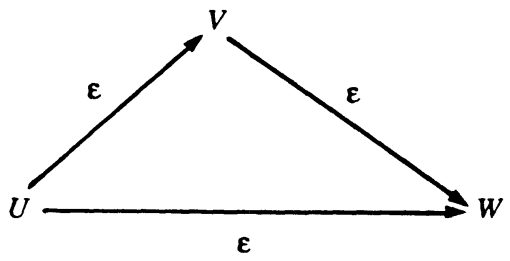

where all $\varepsilon= \pm 1$ are equal.

First, we give the argument for $\varepsilon=+1$.

(I) $S_{1}=R_{2} S_{3}$.

LHS: $S_{1}(k, i) \neq 0$ iff $V_{k} \cap \sigma^{-1}\left(U_{i}\right) \neq \varnothing$.

RHS: $R_{2}(k, p) S_{3}(p, i) \neq 0$ iff $V_{k} \cap W_{p} \neq \varnothing$ and $W_{p} \cap \sigma^{-1}\left(U_{i}\right) \neq \varnothing$.

We claim that RHS is a zero-one matrix. Given $k$, choose some $j$ such that $R_{1}(j, k) \neq 0$. Then $R_{1}(j, k) R_{2}(k, p) S_{3}(p, i)=$ $R_{3}(j, p) S_{3}(p, i)$. Since $R_{3} S_{3}=Q$ is a zero-one matrix, there can be at most one $p$ for which this term is non-zero.

Assume LHS $\neq 0$. We have $W<U \cap \sigma^{-1}(U)<V \cap \sigma^{-1}(U)$. So choose the unique index $p$ with $W_{p} \supset V_{k} \cap \sigma^{-1}\left(U_{i}\right)$. Then $V_{k} \cap$ $W_{p} \neq \varnothing$ and $W_{p} \cap \sigma^{-1}\left(U_{i}\right) \neq \varnothing$ implying RHS $\neq 0$. Conversely, let RHS $\neq 0$. Since $V<W, V_{k} \cap W_{p} \neq \varnothing$ implies $V_{k} \supset W_{p}$. Thus $W_{p} \cap \sigma^{-1}\left(U_{i}\right) \neq \varnothing$ implies $V_{k} \cap \sigma^{-1}\left(U_{i}\right) \neq \varnothing$. This shows LHS $\neq 0$.

(II) $S_{2}=S_{3} R_{1}$.

LHS: $S_{2}(p, k) \neq 0$ iff $W_{p} \cap \sigma^{-1}\left(V_{k}\right) \neq \varnothing$.

RHS: $S_{3}(p, i) R_{1}(i, k) \neq 0$ iff $W_{p} \cap \sigma^{-1}\left(U_{i}\right) \neq \varnothing$ and $U_{i} \cap V_{k} \neq \varnothing$.

Since $U<V$, we have $U_{i} \supset V_{k}$ and thus $i$ is determined by $k$. This shows RHS is a zero-one matrix.

Assume LHS $\neq 0$. Let $i$ be such that $U_{i} \supset V_{k}$. Then $W_{p} \cap$ $\sigma^{-1}\left(U_{i}\right) \neq \varnothing$ and $U_{i} \cap V_{k} \neq \varnothing$ showing that RHS $\neq 0$. Conversely, suppose RHS $\neq 0$. Then $U_{i} \supset V_{k}$ as remarked above. Write

$$
W_{p}=\bigcup U_{a} \cap \sigma^{-1}\left(U_{b}\right), \quad V_{k}=\bigcup U_{i} \cap \sigma^{-1}\left(U_{d}\right) .
$$

The condition $W_{p} \cap \sigma^{-1}\left(U_{l}\right) \neq \varnothing$ implies some $b=i$. Hence,

$$
W_{p} \cap \sigma^{-1}\left(V_{k}\right) \supset U_{a} \cap \sigma^{-1}\left(U_{i}\right) \cap \sigma^{-2}\left(U_{d}\right) \neq \varnothing
$$

for some $a$ and some $d$. Thus LHS $\neq 0$. 
Next, we give the argument for $\varepsilon=-1$. Remember that now $R_{1}=R(W, V), R_{2}=R(V, U), R_{3}=R(W, U)$, etc.

(I) $S_{1}=R_{2} S_{3}$,

LHS: $S_{1}(k, p) \neq 0$ iff $V_{k} \cap \sigma^{-1}\left(W_{p}\right) \neq \varnothing$.

RHS: $R_{2}(k, i) S_{3}(i, p) \neq 0$ iff $V_{k} \cap U_{i} \neq \varnothing$ and $U_{i} \cap \sigma^{-1}\left(W_{p}\right) \neq \varnothing$.

We claim that RHS is a zero-one matrix. Given $k$ and $p$, choose some $q$ such that $R_{1}(q, k) \neq 0$. Then

$$
R_{1}(q, k) R_{2}(k, i) S_{3}(i, p)=R_{3}(q, i) S_{3}(i, p) .
$$

Since $R_{3} S_{3}=Q$ is a zero-one matrix, there can be at most one $i$ for which this term is non-zero.

Assume LHS $\neq 0$. We have $V<U \cap \sigma^{-1}(U)<V \cap \sigma^{-1}(W)$. So choose the unique index $i$ with $U_{i} \supset V_{k} \cap \sigma^{-1}\left(W_{p}\right) \neq \varnothing$. Then $V_{k} \cap U_{i} \neq \varnothing$ and $U_{i} \cap \sigma^{-1}\left(W_{p}\right) \neq \varnothing$ implying RHS $\neq 0$. Conversely, the RHS $\neq 0$. Write

$$
W_{p}=\bigcup \sigma\left(U_{a}\right) \cap U_{b}, \quad V_{k}=\bigcup \sigma\left(U_{c}\right) \cap U_{d} .
$$

Since $V_{k} \cap U_{i} \neq \varnothing$, some $d=i$. Also $U_{i} \cap \sigma^{-1}\left(W_{p}\right) \neq \varnothing$ implies some $a=i$. Therefore

$$
V_{k} \cap \sigma^{-1}\left(W_{p}\right) \supset \sigma\left(U_{c}\right) \cap U_{i} \cap \sigma^{-1}\left(U_{b}\right) \neq \varnothing
$$

for some $c$ and some $b$. Thus LHS $\neq 0$.

(II) $S_{2}=S_{3} R_{1}$.

LHS: $S_{2}(i, k) \neq 0$ iff $U_{i} \cap \sigma^{-1}\left(V_{k}\right) \neq \varnothing$.

RHS: $S_{3}(i, p) R_{1}(p, k) \neq 0$ iff $U_{i} \cap \sigma^{-1}\left(W_{p}\right) \neq \varnothing$ and $W_{p} \cap V_{k} \neq \varnothing$.

We claim that RHS is a zero-one matrix. Given $i$ and $k$, choose some $j$ such that $R_{2}(k, j) \neq 0$. Then

$$
S_{3}(i, p) R_{1}(p, k) R_{2}(k, j)=S_{3}(i, p) R_{3}(p, j) .
$$

Since $S_{3} R_{3}=M$ is a zero-one matrix, there can be at most one $p$ for which this term is non-zero.

Assume LHS $\neq 0$. We have $W<\sigma(U) \cap U<\sigma(U) \cap V$. Let $p$ be the unique index where $W_{p} \supset \sigma\left(U_{i}\right) \cap V_{k} \neq \varnothing$. Then $U_{i} \cap \sigma^{-1}\left(W_{p}\right) \neq \varnothing$ 
and $W_{p} \cap V_{k} \neq \varnothing$. This says RHS $\neq 0$. Conversely, assume RHS $\neq 0$. Since $V<W$, we must have $W_{p} \subset V_{k}$. Hence $U_{i} \cap \sigma^{-1}\left(V_{k}\right) \neq \varnothing$ and LHS $\neq 0$.

Triangle \#2:

This triangle is of the form

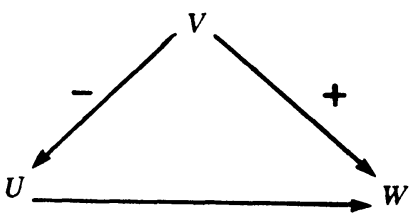

(I) $S_{1}=R_{2} S_{3}$.

LHS: $S_{1}(k, i) \neq 0$ iff $V_{k} \cap \sigma^{-1}\left(U_{i}\right) \neq \varnothing$.

RHS: $R_{2}(k, p) S_{3}(p, i) \neq 0$ iff $V_{k} \cap W_{p} \neq \varnothing$ and $W_{p} \cap \sigma^{-1}\left(U_{i}\right) \neq \varnothing$.

The RHS is a zero-one matrix. To see this, choose $j$ so that $R_{1}(j, k)$

$=1$. Then $R_{1}(j, k) R_{2}(k, p) S_{3}(p, i)=R_{3}(j, p) S_{3}(p, i)$. Since $R_{3} S_{3}=M$ is a zero-one matrix, there is at most one $p$ giving a non-zero term.

Assume LHS $\neq 0$. We have $W<V \cap \sigma^{-1}(V)<V \cap \sigma^{-1}(U)$. So choose $p$ with $W_{p} \supset V_{k} \cap \sigma^{-1}\left(U_{i}\right) \neq \varnothing$. Then $V_{k} \cap W_{p} \neq \varnothing$ and $W_{p} \cap \sigma^{-1}\left(U_{i}\right) \neq \varnothing$ and RHS $\neq 0$. Conversely, suppose RHS $\neq 0$. Then $V_{k} \supset W_{p}$ and $V_{k} \cap \sigma^{-1}\left(U_{i}\right) \neq \varnothing$. This gives LHS $\neq 0$.

(II) $S_{2}=S_{3} R_{1}$.

LHS: $S_{2}(p, k) \neq 0$ iff $W_{p} \cap \sigma^{-1}\left(V_{k}\right) \neq \varnothing$.

RHS: $S_{3}(p, i) R_{1}(i, k) \neq 0$ iff $W_{p} \cap \sigma^{-1}\left(U_{i}\right) \neq \varnothing$ and $U_{i} \cap V_{k} \neq \varnothing$.

The RHS is a zero-one matrix. To see this, let $q$ be any index where $R_{2}(k, q) \neq 0$. Then $S_{3}(p, i) R_{1}(i, k) R_{2}(k, q)=S_{3}(p, i) R_{3}(i, q)$. Since $S_{3} R_{3}=Q$ is zero-one, there is at most one $i$ for which the term is non-zero.

Assume LHS $\neq 0$. We have $U<\sigma(V) \cap V<\sigma(W) \cap V$. Let $i$ be the index for which $U_{i} \supset \sigma\left(W_{p}\right) \cap V_{k} \neq \varnothing$. Then $W_{p} \cap \sigma^{-1}\left(U_{i}\right) \neq \varnothing$ and $U_{i} \cap V_{k} \neq \varnothing$. This shows RHS $\neq 0$. Finally, let RHS $\neq 0$. Then $U_{i} \subset V_{k}$, because $U>V$. Therefore $W_{p} \cap \sigma^{-1}\left(V_{k}\right) \neq \varnothing$ and LHS $\neq 0$.

This completes the argument for the triangles in (2.3). We now show how these combine to prove the RS Identities for (2.1). From 
(2.2) we have the diagram of three triangles

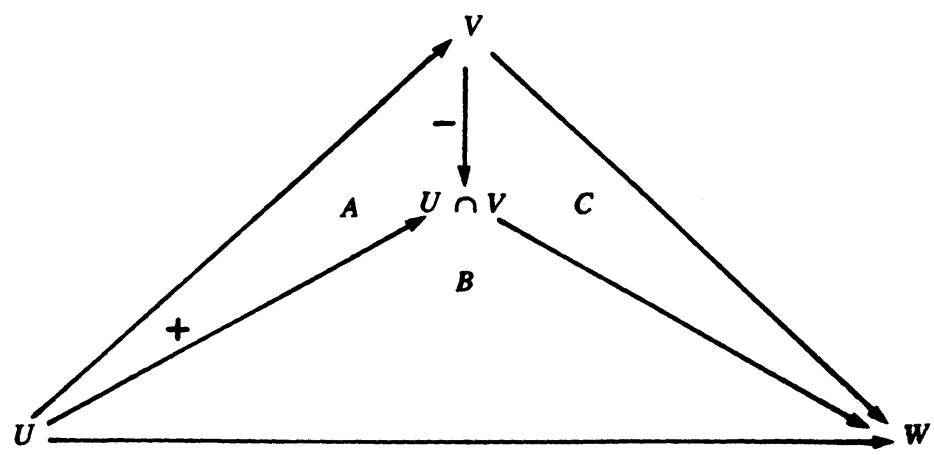

Step 1. We will show the RS Identities for Triangles A, B, and C in (2.4) imply the RS Identities for (2.1).

(I) $S(V, U)=R(V, W) S(W, U)$ ?

Since $U<U \cap V$, the matrix $R(U, U \cap V)$ has exactly one non-zero term in each column. Therefore, the above equation holds provided it holds when multiplied on the right by $R(U, U \cap V)$. We have

$$
S(V, U) R(U, U \cap V)=S(V, U \cap V)
$$

and

$$
\begin{aligned}
& \begin{aligned}
R(V, W) S(W, U) R(U, U \cap V) & =R(V, W) S(W, U \cap V) \\
& =S(V, U \cap V)
\end{aligned} \\
& \text { (II) } S(W, V)=S(W, U) R(U, V) ?
\end{aligned}
$$

$$
\begin{aligned}
S(W, V) & =S(W, U \cap V) R(U \cap V, V) \\
& =S(W, U) R(U, U \cap V) R(U \cap V, V) \\
& =S(W, U) R(U, V) .
\end{aligned}
$$

It remains to check the RS Identities for each of the triangles A, B, C.

Step 2. Triangle A is just Triangle \#1 of (2.3).

Step 3. Triangle $\mathrm{B}$.

From (2.2) and (2.3) we obtain the diagram

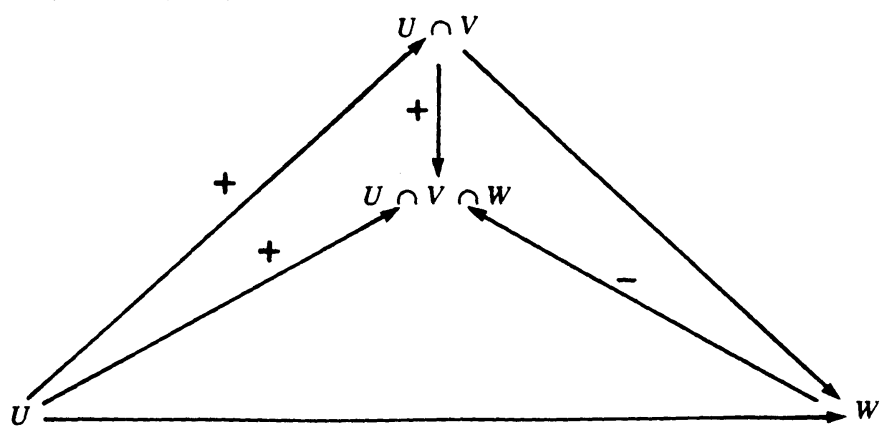


Recall that $U \cap W=U \cap V \cap W$ and that the RS Identities for each of the subtriangles was verified above for (2.3).

(I) $S(U \cap V, U)=R(U \cap V, W) S(W, U)$ ?

$$
\begin{aligned}
S(U \cap V, U) & =R(U \cap V, U \cap V \cap W) S(U \cap V \cap W, U) \\
& =R(U \cap V, U \cap V \cap W) R(U \cap V \cap W, W) S(W, U) \\
& =R(U \cap V, W) S(W, U) .
\end{aligned}
$$

(II) $S(W, U \cap V)=S(W, U) R(U, U \cap V)$ ?

Similarly to (I) of Step 1 this equation holds iff it holds when multiplied on the right by $R(U \cap V, U \cap V \cap W)$. We have

$$
S(W, U \cap V) R(U \cap V, U \cap V \cap W)=S(W, U \cap V \cap W)
$$

and

$$
\begin{aligned}
& S(W, U) R(U, U \cap V) R(U \cap V, U \cap V \cap W) \\
& \quad=S(W, U) R(U, U \cap V \cap W)=S(W, U \cap V \cap W) .
\end{aligned}
$$

Step 4. Triangle C.

From (2.2) and (2.3) we get

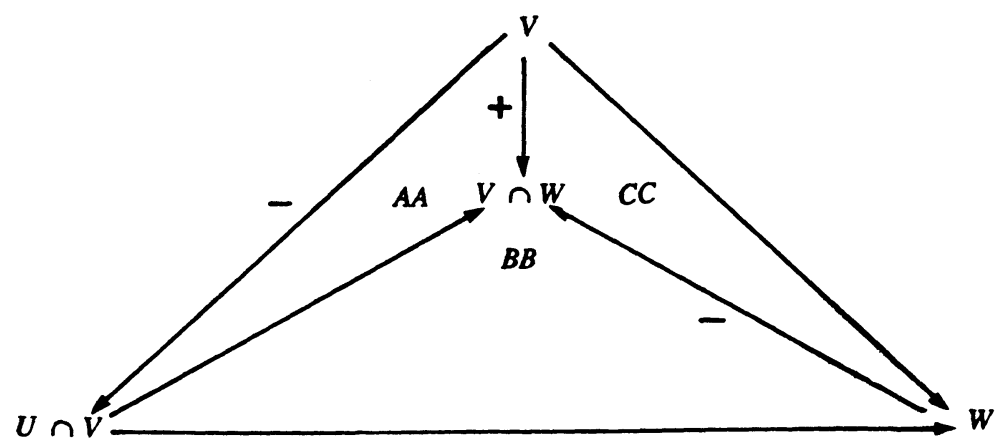

Assuming the RS Identities hold for the Triangles AA, BB, and CC, we now verify the RS Identities for Triangle C. In Step 5 below the RS Identities for AA, BB, CC are checked.

(I) $S(V, U \cap V)=R(V, W) S(W, U \cap V)$ ?

$$
\begin{aligned}
S(V, U \cap V) & =R(V, V \cap W) S(V \cap W, U \cap V) \\
& =R(V, V \cap W) R(V \cap W, W) S(W, U \cap V) \\
& =R(V, W) S(W, U \cap V)
\end{aligned}
$$

(II) $S(W, V)=S(W, U \cap V) R(U \cap V, V)$ ? 
Similarly to above remarks, this equation holds iff it holds when multiplied on the right by $R(V, V \cap W)$. We have

$$
S(W, V) R(V, V \cap W)=S(W, V \cap W)
$$

and

$$
\begin{aligned}
& S(W, U \cap V) R(U \cap V, V) R(V, V \cap W) \\
& \quad=S(W, U \cap V) R(U \cap V, V \cap W)=S(W, V \cap W) .
\end{aligned}
$$

Step 5. Triangles AA, BB, CC

Triangles $\mathrm{AA}$ and $\mathrm{CC}$ are just like Triangles \#2 and \#3 in (2.3). From diagram (2.2) we see that Triangle $\mathrm{BB}$ subdivides into

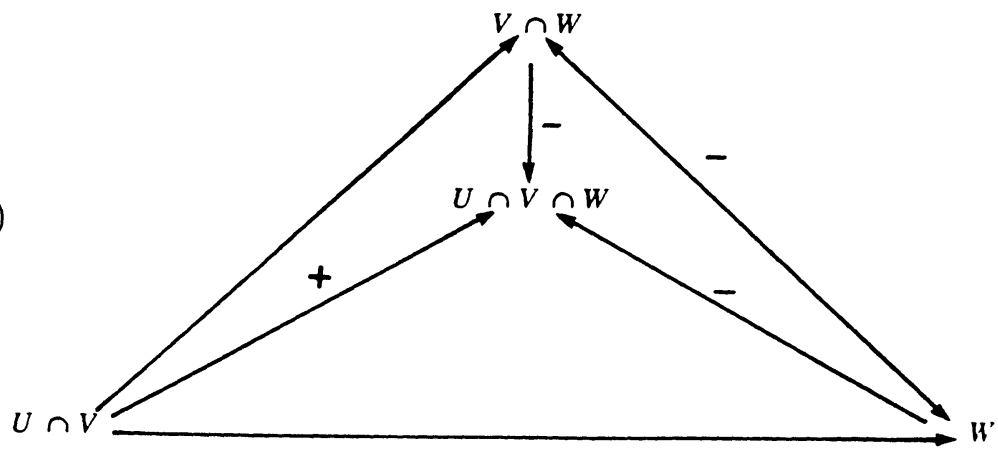

Each of the subtriangles in (2.7) satisfies the RS Identities by Step 1. So proceed as before:

(I) $S(V \cap W, U \cap W)=R(V \cap W, W) S(W, U \cap V)$ ?

The matrix $R(U \cap V \cap W, V \cap W)$ has the property that each row has exactly one non-zero entry. Therefore the above equation holds iff it holds when multiplied on the left by $R(U \cap V \cap W, V \cap W)$. We have

$$
\begin{gathered}
R(U \cap V \cap W, V \cap W) S(V \cap W, U \cap V) \\
=S(U \cap V \cap W, U \cap V)
\end{gathered}
$$

and

$$
\begin{aligned}
& R(U \cap V \cap W, V \cap W) R(V \cap W, W) S(W, U \cap V) \\
& \quad=R(U \cap V \cap W, W) S(W, U \cap V)=S(U \cap V \cap W, U \cap V) . \\
& (\mathrm{II}) \quad S(W, V \cap W)=S(W, U \cap V) R(U \cap V, V \cap W) ? \\
& S(W, V \cap W)=S(W, U \cap V \cap W) R(U \cap V \cap W, V \cap W) \\
& \quad=S(W, U \cap V) R(U \cap V, U \cap V \cap W) R(U \cap V \cap W, V \cap W) \\
& \quad=S(W, U \cap V) R(U \cap V, V \cap W) .
\end{aligned}
$$

This finally completes the proof of the RS Triangle Identities. 
3. The isomorphisms $\Phi_{A}$ and $\Theta_{A}$. Our first goal is to give a formula in (3.5) and (3.8) for the homomorphism

$$
\Phi_{A}: \operatorname{Aut}\left(\sigma_{A}\right) \rightarrow \pi_{1}(\mathrm{RS}(\mathscr{E}), A) .
$$

If the square matrices $A$ and $B$ lie in the same component of $\mathrm{RS}(\mathscr{E})$, let

$$
\pi_{1}(\mathrm{RS}(\mathscr{E}) ; A, B)
$$

denote the set of homotopy classes of paths starting at $A$ and ending at $\mathrm{B}$. Concatenation of paths gives a pairing

$$
\pi_{1}(\mathrm{RS}(\mathscr{E}) ; A, B) \times \pi_{1}(\operatorname{RS}(\mathscr{E}) ; B, C) \rightarrow \pi_{1}(\operatorname{RS}(\mathscr{E}) ; A, C)
$$

denoted by "**. When $A=B$ we just have the fundamental group $\pi_{1}(\operatorname{RS}(\mathscr{E}), A)$.

Let $\operatorname{Isom}\left(\sigma_{A}, \sigma_{B}\right)$ denote the set of conjugacies $\alpha:\left(X_{A}, \sigma_{A}\right) \rightarrow$ $\left(X_{B}, \sigma_{B}\right)$. Suppose $U \rightarrow V$ in $P_{A}$ with $P=M(U)$ and $Q=M(V)$, and let $R$ and $S$ be defined as in $\S 1$ giving a strong shift equivalence $(R, S): P \rightarrow Q$. Let $\alpha$ be in $\operatorname{Isom}\left(\sigma_{A}, \sigma_{B}\right)$. Let $U^{\prime}=\alpha(U)$ and $V^{\prime}=\alpha(V)$ in $P_{B}$. Let $P^{\prime}=M\left(U^{\prime}\right)$ and $Q^{\prime}=M\left(V^{\prime}\right)$. We have $U^{\prime} \rightarrow V^{\prime}$ in $P_{B}$. Let $R^{\prime}$ and $S^{\prime}$ be the corresponding matrices giving a strong shift equivalence from $P^{\prime}$ to $Q^{\prime}$. These matrices satisfy the identities

$$
\begin{array}{ll}
P^{\prime}=\alpha P \alpha^{-1}, & Q^{\prime}=\alpha Q \alpha^{-1}, \\
R^{\prime}=\alpha R \alpha^{-1}, & S^{\prime}=\alpha S \alpha^{-1},
\end{array}
$$

which translate into the following diagrams of triangles in $\operatorname{RS}(\mathscr{E})$ :
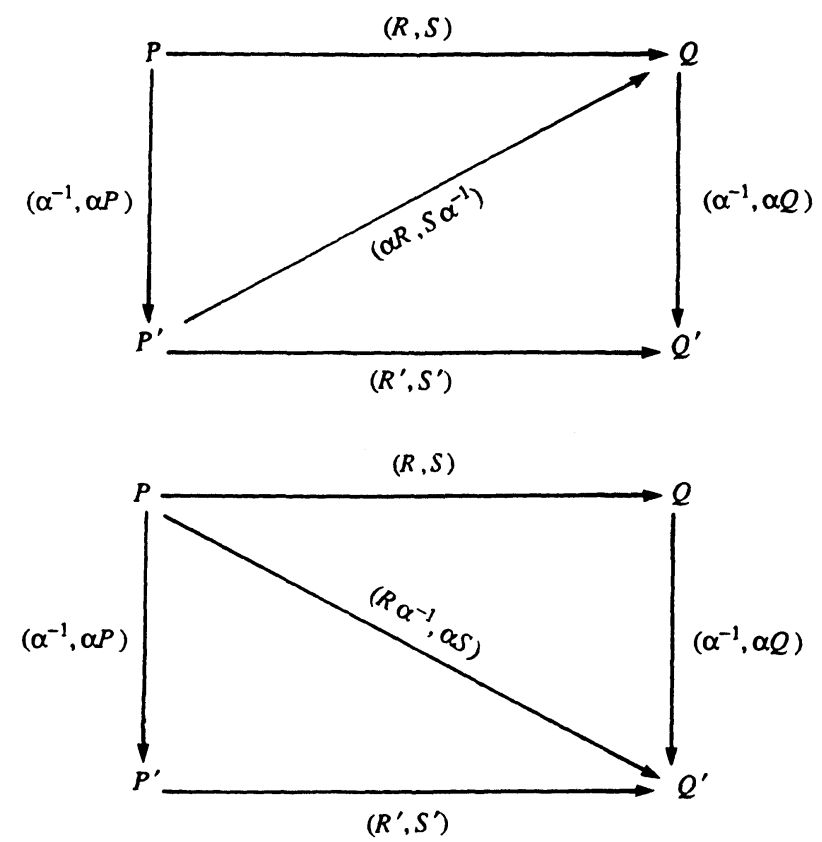
Incidentally, these two diagrams form the boundary of the tetrahedron $\left\langle P, P^{\prime}, Q, Q^{\prime}\right\rangle$ in $\mathrm{RS}(\mathscr{E})$.

LEMMA 3.3. (a) $\gamma(1, A)=1$ in $\pi_{1}(R S(\mathscr{E}), A)$.

(b) If $\gamma \in \pi_{1}(R S(\mathscr{E}) ; A, B)$, then $\gamma(A, 1) * \gamma=\gamma * \gamma(B, 1)$.

(c) $\gamma(R, S) * \gamma(S, R)=\gamma(A, 1)$.

(d) $\gamma\left(\alpha^{-1}, \alpha P\right)^{-1}=\gamma\left(\alpha, P \alpha^{-1}\right)$.

Proof of (a). The triangle

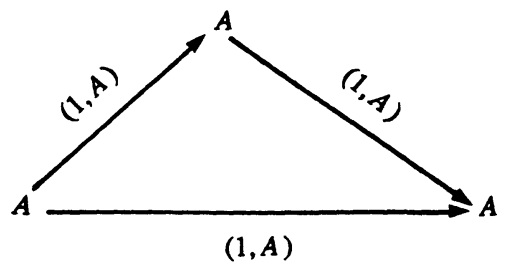

shows that $\gamma(1, A) * \gamma(1, A)=\gamma(1, A)$. Now cancel.

Proof of (b) and (c). Since $\gamma$ is a product of paths $\gamma(R, S)$ and their inverses, it suffices to consider the case $\gamma=\gamma(R, S)$. The formula (b) then follows from the diagram

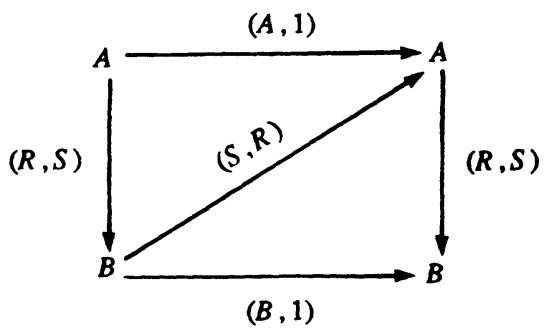

The formula (c) is a consequence of the top triangle in this diagram.

Proof of $(\mathrm{d})$. This follows from the triangle

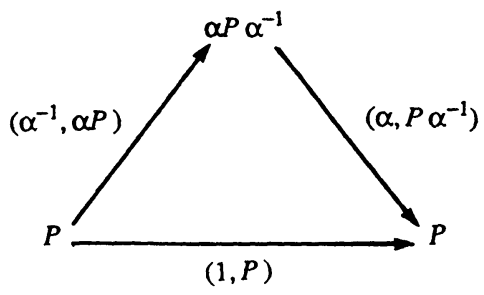


Proposition 3.4. Let $U$ and $V$ be in $P_{A}$ with $P=M(U)$ and $Q=M(V)$. Then there is a well defined path $\Gamma(U, V)$ in $\pi_{1}(\mathrm{RS}(\mathscr{E}) ; P, Q)$ such that

$$
\begin{aligned}
\Gamma(U, U) & =1, \\
\Gamma(U, W) & =\Gamma(U, V) * \Gamma(V, W) .
\end{aligned}
$$

Moreover, if $\alpha \in \operatorname{Isom}\left(\sigma_{A}, \sigma_{B}\right)$, then

$$
\begin{aligned}
\Gamma(\alpha(U), \alpha(V)) & =\gamma\left(\alpha^{-1}, \alpha P\right)^{-1} * \Gamma(U, V) * \gamma\left(\alpha^{-1}, \alpha Q\right) \\
& =\gamma\left(\alpha, P \alpha^{-1}\right) * \Gamma(U, V) * \gamma\left(\alpha^{-1}, \alpha Q\right) .
\end{aligned}
$$

Proof. For the special case $U \rightarrow V$ in $P_{A}$, define $\Gamma(U, V)=$ $\gamma(R, S)$. In general, choose a path from $U$ to $V$ in $P_{A}$ which is concatenation of edges $\left\langle U_{i-1}, U_{i}\right\rangle^{\varepsilon(i)}$ for $i=1, \ldots, n$ where $\varepsilon(i)= \pm 1$. Then define

(3.5) $\Gamma(U, V)=\Gamma\left(U_{0}, U_{1}\right)^{\varepsilon(1)} * \Gamma\left(U_{1}, U_{2}\right)^{\varepsilon(2)} * \cdots * \Gamma\left(U_{n-1}, U_{n}\right)^{\varepsilon(n)}$.

From the definition of $\Gamma$, we see that $\Gamma(U, U)=\gamma(1, A)=1$ by (a) of (3.3). It is also clear that $\Gamma(U, V) * \Gamma(V, W)=\Gamma(U, W)$ provided $\Gamma$ is independent of the path chosen in $P_{A}$ from $U$ to $V$. But this follows immediately from the RS Triangle Identities (1.2) and simple connectivity of $P_{A}$ proved in [8]. The formula for $\Gamma(\alpha(U), \alpha(V))$ follows from the diagram (3.1) and (d) of (3.3).

Proposition 3.6. There is a map $\Phi=\Phi(A, B)$ from $\operatorname{Isom}\left(\sigma_{A}, \sigma_{B}\right)$ to $\pi_{1}(R S(\mathscr{E}) ; A, B)$ such that if $\alpha \in \operatorname{Isom}\left(\sigma_{A}, \sigma_{B}\right)$ and $\beta \in$ $\operatorname{Isom}\left(\sigma_{B}, \sigma_{C}\right)$ then

$$
\Phi(\beta \alpha)=\Phi(\alpha) * \Phi(\beta) .
$$

Considering $\sigma_{A} \in \operatorname{Aut}\left(\sigma_{A}\right)=\operatorname{Isom}\left(\sigma_{A}, \sigma_{A}\right)$ we have

$$
\Phi\left(\sigma_{A}\right)=\gamma(A, 1) \text {. }
$$

From this result we then obtain the homomorphism

$$
\Phi_{A}: \operatorname{Aut}\left(\sigma_{A}\right) \rightarrow \pi_{1}(\operatorname{RS}(\mathscr{E}), A)
$$

by taking $A=B$ and setting $\Phi_{A}(\alpha)=\Phi\left(\alpha^{-1}\right)$.

Proof. Let $\alpha \in \operatorname{Isom}\left(\alpha_{A}, \sigma_{B}\right)$. We then have

$$
\left(\alpha^{-1}, \alpha A\right): A=M\left(U^{A}\right) \rightarrow M\left(\alpha\left(U^{A}\right)\right)=\alpha A \alpha^{-1} \text {. }
$$

Define

$$
\Phi(\alpha)=\gamma\left(\alpha^{-1}, \alpha A\right) * \Gamma\left(\alpha\left(U^{A}\right), U^{B}\right) .
$$


Now let $\alpha \in \operatorname{Isom}\left(\sigma_{A}, \sigma_{B}\right)$ and $\beta \in \operatorname{Isom}\left(\sigma_{B}, \sigma_{C}\right)$. Then

$$
\begin{aligned}
\Phi(\beta \alpha)= & \gamma\left(\alpha^{-1} \beta^{-1}, \alpha \beta A\right) * \Gamma\left(\beta \alpha\left(U^{A}\right), U^{C}\right) \\
= & \gamma\left(\alpha^{-1}, \alpha A\right) * \gamma\left(\beta^{-1}, \alpha \beta A \alpha^{-1}\right) * \Gamma\left(\beta \alpha\left(U^{A}\right), \beta\left(U^{B}\right)\right) \\
& * \Gamma\left(\beta\left(U^{B}\right), U^{C}\right) .
\end{aligned}
$$

From (3.4) we see that

$$
\begin{aligned}
& \Gamma\left(\beta \alpha\left(U^{A}\right), \beta\left(U^{B}\right)\right) \\
& \quad=\gamma\left(\beta^{-1}, \alpha \beta A \alpha^{-1}\right)^{-1} * \Gamma\left(\alpha\left(U^{A}\right), U^{B}\right) * \gamma\left(\beta^{-1}, \beta B\right) .
\end{aligned}
$$

Substituting and simplifying gives

$$
\begin{aligned}
\Phi(\beta \alpha) & =\gamma\left(\alpha^{-1}, \alpha A\right) * \Gamma\left(\alpha\left(U^{A}\right), U^{B}\right) * \gamma\left(\beta^{-1}, \beta B\right) * \Gamma\left(\beta\left(U^{B}\right), U^{C}\right) \\
& =\Phi(\alpha) * \Phi(\beta) .
\end{aligned}
$$

To compute $\Phi\left(\sigma_{A}\right)$, recall that $\sigma_{A}\left(U^{A}\right) \rightarrow U^{A}$. We then have the triangle

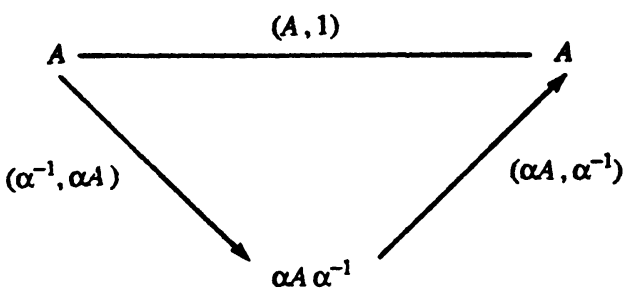

where $\alpha$ is the bijection between the states $U^{A}$ and the states of $\sigma_{A}\left(U^{A}\right)$ induced by $\sigma_{A}$. This shows

$$
\Phi\left(\sigma_{A}\right)=\gamma\left(\alpha^{-1}, \alpha A\right) * \Gamma\left(\sigma_{A}\left(U^{A}\right), U^{A}\right)=\gamma(A, 1) .
$$

This completes the construction of $\Phi_{A}$. Next consider the homomorphism

$$
\Theta_{A}: \pi_{1}(\mathrm{RS}(\mathscr{E}), A) \rightarrow \operatorname{Aut}\left(\sigma_{A}\right) .
$$

The formula for $\Theta_{A}$ was given in (1.8), and as discussed in $\S 1$ it only remains to give the

Proof of (1.9). Suppose that $(R, S): A \rightarrow B$. The equation $c(R, S) c(S, R)=\sigma_{A}$ implies $c(R, S)^{-1}=\sigma_{A}^{-1} c(S, R)$. If $y=$ $\left\{y_{n}\right\} \in X_{B}$, we can therefore characterize $x=c(R, S)^{-1}(y)$ to be the unique point $x=\left\{x_{n}\right\} \in X_{A}$ such that

$$
S\left(y_{n-1}, x_{n}\right) R\left(x_{n}, y_{n}\right)=1
$$

for all $n$. 
First we will show that the RS Triangle Identities imply

$$
c\left(R_{2}, S_{2}\right) c\left(R_{1}, S_{1}\right)=c\left(R_{3}, S_{3}\right) .
$$

Let $x=\left\{x_{n}\right\} \in X_{M}$ with $y=c\left(R_{1}, S_{1}\right)(x)=\left\{y_{n}\right\}$ and $z=$ $c\left(R_{2}, S_{2}\right)(y)=\left\{z_{n}\right\}$. Let $w=c\left(R_{3}, S_{3}\right)^{-1}(z)=\left\{w_{n}\right\}$. We want to show that $x_{n}=w_{n}$ for all $n$. We know that $w_{n}$ is the unique state satisfying

$$
S_{3}\left(z_{n-1}, w_{n}\right) R_{3}\left(w_{n}, z_{n}\right)=1 .
$$

Let $k$ be the unique state such that

$$
S_{3}\left(z_{n-1}, w_{n}\right) R_{1}\left(w_{n}, k\right) R_{2}\left(k, z_{n}\right)=S_{2}\left(z_{n-1}, k\right) R_{2}\left(k, z_{n}\right)=1 .
$$

Then $k=y_{n}$. We also have

$$
R_{3}\left(x_{n-1}, z_{n-1}\right)=R_{1}\left(x_{n-1}, y_{n-1}\right) R_{2}\left(y_{n-1}, z_{n-1}\right)=1 \text {. }
$$

Thus

$$
R_{2}\left(y_{n-1}, z_{n-1}\right) S_{3}\left(z_{n-1}, w_{n}\right)=S_{1}\left(y_{n-1}, w_{n}\right)=1
$$

and

$$
S_{1}\left(y_{n-1}, w_{n}\right) R_{1}\left(w_{n}, y_{n}\right)=1 \text {. }
$$

We conclude that $w_{n}=x_{n}$.

For the converse, remember that each matrix in $\mathscr{E}$ has at least one non-zero entry in each row and in each column.

The equation $S_{3} R_{1}=S_{2}$ : The left hand side LHS is a zero-one matrix, because $S_{3} R_{1} R_{2}=S_{3} R_{3}=Q$ is zero-one. Fix a pair of indices $(p, l)$ such that $S_{2}(p, l)=1$. Choose points $z=\left\{z_{n}\right\} \in X_{Q}$ and $y=\left\{y_{n}\right\} \in X_{P}$ such that $z_{0}=p, y_{1}=l$, and $z=c\left(R_{2}, S_{2}\right)(y)$. Then choose $x=\left\{x_{n}\right\} \in X_{M}$ with $y=c\left(R_{1}, S_{1}\right)(x)$. In particular, we have $z=c\left(R_{2}, S_{2}\right) c\left(R_{1}, S_{1}\right)(x)=c\left(R_{3}, S_{3}\right)(x)$. Then

$$
1=S_{3}\left(z_{0}, x_{1}\right) R_{3}\left(x_{1}, z_{1}\right)=S_{3}\left(z_{0}, x_{1}\right) R_{1}\left(x_{1}, y_{1}\right) R_{2}\left(y_{1}, z_{1}\right)
$$

and $S_{3}\left(p, x_{1}\right) R_{1}\left(x_{1}, l\right)=1$. Thus LHS $\neq 0$. On the other hand, suppose for an index $i$ that $S_{3}(p, i) R_{1}(i, l)=1$. We must show $S_{2}(p, l)=1$. Choose a point $z$ in $X_{Q}$ with $z_{0}=p$ and $R_{2}\left(l, z_{1}\right)=$ 1. Then choose a point $x$ in $X_{M}$ with $z=c\left(R_{3}, S_{3}\right)(x)$ and $x_{1}=i$. Consider $y=c\left(R_{1}, S_{1}\right)(x)$. Then $z=c\left(R_{2}, S_{2}\right)(y)$. We claim that $y_{1}=l$. Recall $R_{1}\left(x_{1}, y_{1}\right) R_{2}\left(y_{1}, z_{1}\right)=1$. But $l$ is the unique state with $R_{3}\left(i, z_{1}\right)=R_{1}(i, l) R_{2}\left(l, z_{1}\right)=1$. Hence $y_{1}=l$. Since $y=c\left(R_{2}, S_{2}\right)^{-1}(z)$, we must have $S_{2}(p, l) R_{2}\left(l, z_{1}\right)=1$ and so $S_{2}(p, l)=1$. 
The equation $R_{2} S_{3}=S_{1}$ : The left hand side LHS is a zero-one matrix, because $R_{1} R_{2} S_{3}=R_{3} S_{3}=M$ is zero-one. Suppose RHS $\neq 0$ and fix a pair of indices $(k, i)$ such that $S_{1}(k, i)=1$. Choose $x$ in $X_{M}$ and $y$ in $X_{P}$ with $x_{1}=i, y_{0}=k$, and $y=c\left(R_{1}, S_{1}\right)(x)$. Let $z=c\left(R_{2}, S_{2}\right)(y)=c\left(R_{3}, S_{3}\right)(x)$. Then $R_{3}\left(x_{0}, z_{0}\right)=$ $R_{1}\left(x_{0}, y_{0}\right) R_{2}\left(y_{0}, z_{0}\right)=1$ and $S_{3}\left(z_{0}, x_{1}\right)=1$. Therefore $R_{2}\left(k, z_{0}\right) S_{3}\left(z_{0}, i\right)=1$, which says LHS $\neq 0$. Conversely, assume LHS $\neq 0$ and choose a state $p$ with $R_{2}(k, p) S_{3}(p, i)=1$. Choose an $x$ in $X_{M}$ such that $x_{1}=i$ and $R_{1}\left(x_{0}, k\right)=1$. Then $R_{1}\left(x_{0}, k\right) R_{2}(k, p) S_{3}(p, i)=R_{3}\left(x_{0}, p\right) S_{3}(p, i)=1$. Let $y=$ $c\left(R_{1}, S_{1}\right)(x)$ and $z=c\left(R_{2}, S_{2}\right)(y)=c\left(R_{3}, S_{3}\right)(x)$. Then $z_{0}=p$ and $y_{0}$ must satisfy $R_{1}\left(x_{0}, y_{0}\right) S_{1}\left(y_{0}, x_{1}\right)=1$. We also have $R_{1}\left(x_{0}, y_{0}\right) R_{2}\left(y_{0}, z_{0}\right) S_{3}\left(z_{0}, x_{1}\right)=1$. Thus $R_{1}\left(x_{0}, y_{0}\right) R_{2}\left(y_{0}, z_{0}\right)=$ 1 , so $y_{0}=k$. This gives $S_{1}(k, i)=1$.

\section{Proof that $\Phi_{A}$ and $\Theta_{A}$ are isomorphisms.}

\section{Step 1. $\Theta_{A} \Phi_{A}=$ Id .}

Let $U=\left\{U_{k}\right\}$ be Markov partition in $P_{A}$ with $P=M(U)$. We have the well-known isomorphism $I=I\left(U, U^{A}\right)$ in $\operatorname{Isom}\left(\sigma_{A}, \sigma_{P}\right)$ defined on $x$ in $X_{A}$ by the condition

$$
I(x)_{n}=k \quad \text { if and only if } \sigma_{A}^{n}(x) \in U_{k} .
$$

See [8] for example. Now consider an isomorphism $\alpha \in \operatorname{Isom}\left(\sigma_{A}, \sigma_{B}\right)$ and let $U \in P_{A}$ and $V \in P_{B}$ where $\alpha(U)=V$. Let $U=\left\{U_{e}\right\}$ and $V=\left\{V_{f}\right\}$ where $e$ and $f$ run through indexing sets $E$ and $F$ respectively. The homeomorphism $\alpha$ induces a bijection from $E$ to $F$. Let $P=M(U)$ and $Q=M(V)$. Then $Q=\alpha P \alpha^{-1}$ and $\left(\alpha^{-1}, \alpha P\right): P \rightarrow Q$.

\section{LEMMA 3.10. $I\left(V, U^{B}\right) \alpha=c\left(\alpha^{-1}, \alpha P\right) I\left(U, U^{A}\right)$.}

Proof. Let $I_{U}=I\left(U, U^{A}\right), I_{V}=I\left(V, U^{B}\right)$, and $c=c\left(\alpha^{-1}, \alpha P\right)$. Remember that $c$ is the one-block map taking $y=\left\{y_{n}\right\}$ with $y_{n} \in E$ to $\alpha(y)=\left\{\alpha\left(y_{n}\right)\right\}$ with $\alpha\left(y_{n}\right) \in F$. Since $\alpha, c, I_{U}$, and $I_{V}$ are shift commuting, it suffices to show that

$$
I_{V}(\alpha(x))_{0}=c\left(I_{U}(x)\right)_{0}
$$

for all $x \in X_{A}$. Let $y=I_{U}(x)=\left\{y_{n}\right\}$ with $y_{n} \in E$. Let $e=$ $y_{0}$. Then $c\left(I_{U}(x)\right)_{0}=f$ if and only if $\alpha(e)=f$. That is, if and only if $\alpha\left(U_{e}\right)=V_{f}$ where $x \in U_{e}$. On the other hand, suppose 
$h=I_{V}(\alpha(x))_{0}$. Then $\alpha(x) \in V_{h}$. Let $g \in E$ be the unique index where $x \in U_{g}$ with $\alpha\left(U_{g}\right)=V_{h}$. Then $e=g$ and so $f=h$.

Let $U \rightarrow V$ in $P_{A}$. Let $R=R(U, V)$ and $S=S(V, U)$.

LEMMA 3.11. $I\left(V, U^{A}\right)=c(R, S) I\left(U, U^{A}\right)$.

Since $I\left(U^{A}, U^{A}\right)=1$, we get $I\left(U, U^{A}\right)=c(R, S)$ whenever $U^{A} \rightarrow U$ in $P_{A}$.

Proof. Let $I=I\left(U, U^{A}\right), J=I\left(V, U^{A}\right)$, and $c=c(R, S)$. It suffices to show that

$$
J(x)_{0}=c I(x)_{0}
$$

for all $x \in X_{A}$. Let $y=I(x)=\left\{y_{n}\right\}$ with $y_{0}=k$ and $y_{1}=l$. This means $x \in U_{k}$ and $\sigma_{A}(x) \in U_{l}$, and consequently $U_{k} \cap \sigma_{A}^{-1}\left(U_{l}\right) \neq \varnothing$. Using $V<U \cap \sigma_{A}^{-1}(U)$, let $V_{b}$ be the unique set in $V$ with $V_{b} \supset$ $U_{k} \cap \sigma_{A}^{-1}\left(U_{l}\right)$. Then $J\left(x_{0}\right)=b$. But also we have $U_{k} \cap V_{b} \neq \varnothing$. Equivalently, $R(k, b) S(b, l)=1$, which implies $c(y)_{0}=b$.

We are now ready to prove $\Theta_{A} \Phi_{A}=$ Id. Recall that $\Phi_{A}(\alpha)=$ $\Phi\left(\alpha^{-1}\right)$. We will show more generally for $\alpha \in \operatorname{Isom}\left(\sigma_{A}, \sigma_{B}\right)$ that $\Theta \Phi(\alpha)=\alpha^{-1}$ where

$$
\Phi: \operatorname{Isom}\left(\sigma_{A}, \sigma_{B}\right) \rightarrow \pi_{1}(\mathrm{RS}(\mathscr{E}) ; A, B)
$$

is defined by (3.8), and

$$
\Theta: \pi_{1}(\operatorname{RS}(\mathscr{E}) ; A, B) \rightarrow \operatorname{Isom}\left(\sigma_{B}, \sigma_{A}\right)
$$

is defined on

$$
\gamma=\prod_{i=1}^{n} \gamma\left(R_{i}, S_{i}\right)^{\varepsilon_{i}}
$$

by the formula

$$
\Theta(\gamma)=\prod_{i=1}^{n} c\left(R_{i}, S_{i}\right)^{-\varepsilon_{i}} .
$$

Let $\alpha \in \operatorname{Isom}\left(\sigma_{A}, \sigma_{B}\right)$ and let

$$
\alpha\left(U^{A}\right)=V_{0} \rightarrow V_{1} \leftarrow \cdots \rightarrow V_{n-1} \leftarrow V_{n}=U^{B}
$$

be the path in $P_{B}$ as in the definition (3.8). For $0 \leq k \leq n$, let $A_{k}=M\left(V_{k}\right)$. For $1 \leq k \leq n, R_{k}=R\left(V_{k-1}, V_{k}\right), S_{k}=S\left(V_{k}, V_{k-1}\right)$, 
and $\varepsilon_{k}=+1$ when $V_{k-1} \rightarrow V_{k}$, and let $R_{k}=R\left(V_{k}, V_{k-1}\right), S_{k}=$ $S\left(V_{k-1}, V_{k}\right)$, and $\varepsilon_{k}=-1$ when $V_{k} \rightarrow V_{k-1}$. Then (3.10) and (3.11) show that

$$
\begin{aligned}
\alpha^{-1} & =c\left(\alpha^{-1}, \alpha A\right)^{-1} \prod_{k=1}^{n} c\left(R_{k}, S_{k}\right)^{-\varepsilon_{k}} \\
& =\Theta\left(\gamma\left(\alpha^{-1}, \alpha A\right) \prod_{k=1}^{n} \gamma\left(R_{k}, S_{k}\right)^{\varepsilon_{k}}\right)=\Theta \Phi(\alpha) .
\end{aligned}
$$

Step II: $\Phi_{A} \Theta_{A}=\mathrm{Id}$.

Assume $(R, S): A \rightarrow B$ and let $c=c(R, S): X_{A} \rightarrow X_{B}$. Let $U^{A}=\left\{U_{i}^{A}\right\}$ and $U^{B}=\left\{U_{k}^{B}\right\}$ be the standard Markov partitions for $\sigma_{A}$ and $\sigma_{B}$ respectively. Let $U=\left\{U_{k}\right\}=\left\{c^{-1}\left(U_{k}^{B}\right)\right\}=c^{-1}\left(U^{B}\right)$ and $V=\left\{V_{i}\right\}=\left\{c\left(U_{i}^{A}\right)\right\}=c\left(U^{A}\right)$.

\section{LeMma 3.13. $U^{A} \rightarrow U$ in $P_{A}$ and $V \rightarrow U^{B}$ in $P_{B}$.}

Proof. It clearly suffices to verify $U^{A} \rightarrow U$. The definition of $c(R, S)$ shows that $U_{k}$ consists of those points $x$ in $X_{A}$ such that $R\left(x_{0}, k\right) S\left(k, x_{1}\right)=1$. In other words,

$$
U_{k}=\bigcup U_{i}^{A} \cap \sigma_{A}^{-1}\left(U_{j}^{A}\right)
$$

where the pairs $(i, j)$ run over those states with $R(i, k) S(k, j)=1$. For a given set $U_{i}^{A} \cap \sigma_{A}^{-1}\left(U_{j}^{A}\right)$ in $U^{A} \cap \sigma_{A}^{-1}\left(U^{A}\right)$, let $k$ be the unique state such that $R(i, k) S(k, j)=1$. Then $U_{i}^{A} \cap \sigma_{A}^{-1}\left(U_{j}^{A}\right) \subset U_{k}$. This shows that $U^{A} \rightarrow U^{A} \cap U$. Next we verify $U \rightarrow U^{A} \cap U$. Fix a pair of states $(k, l)$ and write

$$
U_{k}=\bigcup U_{a}^{A} \cap \sigma_{A}^{-1}\left(U_{b}^{A}\right), \quad U_{l}=\bigcup U_{c}^{A} \cap \sigma_{A}^{-1}\left(U_{d}^{A}\right) .
$$

Then

$$
\sigma_{A}\left(U_{k}\right) \cap U_{l}=\bigcup \sigma_{A}\left(U_{a}^{A}\right) \cap U_{b}^{A} \cap U_{c}^{A} \cap \sigma_{A}^{-1}\left(U_{d}^{A}\right)
$$

where each non-empty term must have $b=c, R(a, k) S(k, b)=1$, and $R(c, l) S(l, d)=1$. Then $R(k, b) S(b, l)=1$, and so only one 
$b$ can occur. The expression becomes

$$
\sigma_{A}\left(U_{k}\right) \cap U_{l}=\bigcup \sigma_{A}\left(U_{a}^{A}\right) \cap U_{b}^{A} \cap \sigma_{A}^{-1}\left(U_{d}^{A}\right) \subset U_{b}^{A} \cap U_{l}
$$

This completes the proof of (3.13).

Let $\alpha \in \operatorname{Isom}\left(\sigma_{A}, \sigma_{B}\right)$ be of the form $\alpha=c(R, S)$ corresponding to $(R, S): A \rightarrow B$ and let $f \in \operatorname{Isom}\left(\sigma_{B}, \sigma_{C}\right)$. Let $U=f \alpha\left(U^{A}\right)$ and $V=f\left(U^{B}\right)$. From (3.13) we see that $U \rightarrow V$ in $P_{B}$. Let $A^{\prime}=M(U), B^{\prime}=M(V), R^{\prime}=R(U, V)$, and $S^{\prime}=S(V, U)$. Then

$$
\begin{array}{ll}
A^{\prime}=f \alpha A \alpha^{-1} f^{-1}, & B^{\prime}=f B f^{-1}, \\
R^{\prime}=f \alpha R f^{-1}, & S^{\prime}=f S \alpha^{-1} f^{-1},
\end{array}
$$

and moreover there is the following diagram of triangles in $\operatorname{RS}(\mathscr{E})$ :

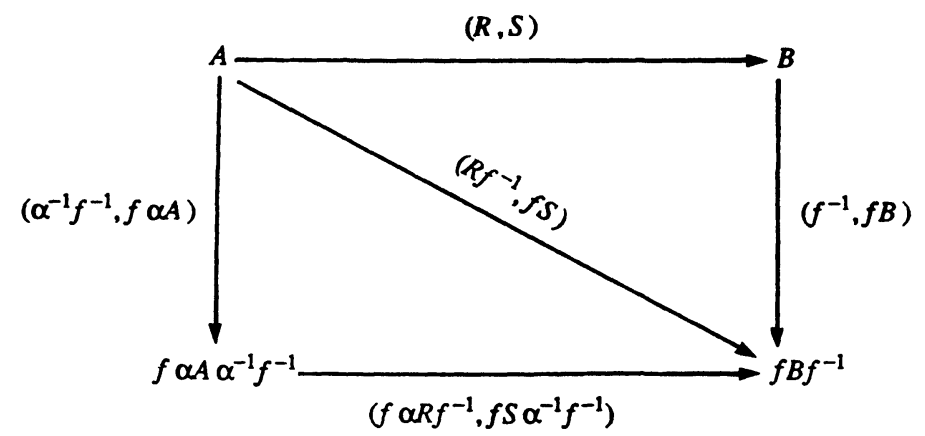

Similarly to Step I we will show that $\Phi \Theta\left(\gamma^{-1}\right)=\gamma$ for any path $\gamma=\prod \gamma\left(R_{k}, S_{k}\right)^{\varepsilon_{k}}$ from $A=A_{0}$ to $B=A_{n}$ in $\pi_{i}(\operatorname{RS}(\mathscr{E}) ; A, B)$. For $0 \leq k \leq n-1$, let $\alpha_{k} \in \operatorname{Isom}\left(\sigma_{A_{k}}, \sigma_{B}\right)$ be given by the formula

$$
\alpha_{k}=c\left(R_{n}, S_{n}\right)^{\varepsilon_{n}} \cdots c\left(R_{k+1}, S_{k+1}\right)^{\varepsilon_{k+1}} .
$$

Let $\alpha_{n}=\mathrm{id}$. Remember that our convention is to read composition of homeomorphisms between spaces from right to left. Let $V_{k}=$ $\alpha_{k}\left(U^{A_{k}}\right)$ in $P_{B}$ for $0 \leq k \leq n-1$ and let $V_{n}=U^{B}$. From (3.1) we know that $V_{k-1} \rightarrow V_{k}$ if $\varepsilon_{k}=+1$ and $V_{k} \rightarrow V_{k-1}$ if $\varepsilon_{k}=-1$. Let $B_{k}=M\left(V_{k}\right)$. Then $B_{k}=\alpha_{k} A_{k} \alpha_{k}^{-1}$. From (3.14) we have the 
following two diagrams corresponding respectfully to the parities $\varepsilon_{k}=$ +1 and $\varepsilon_{k}=-1$ :
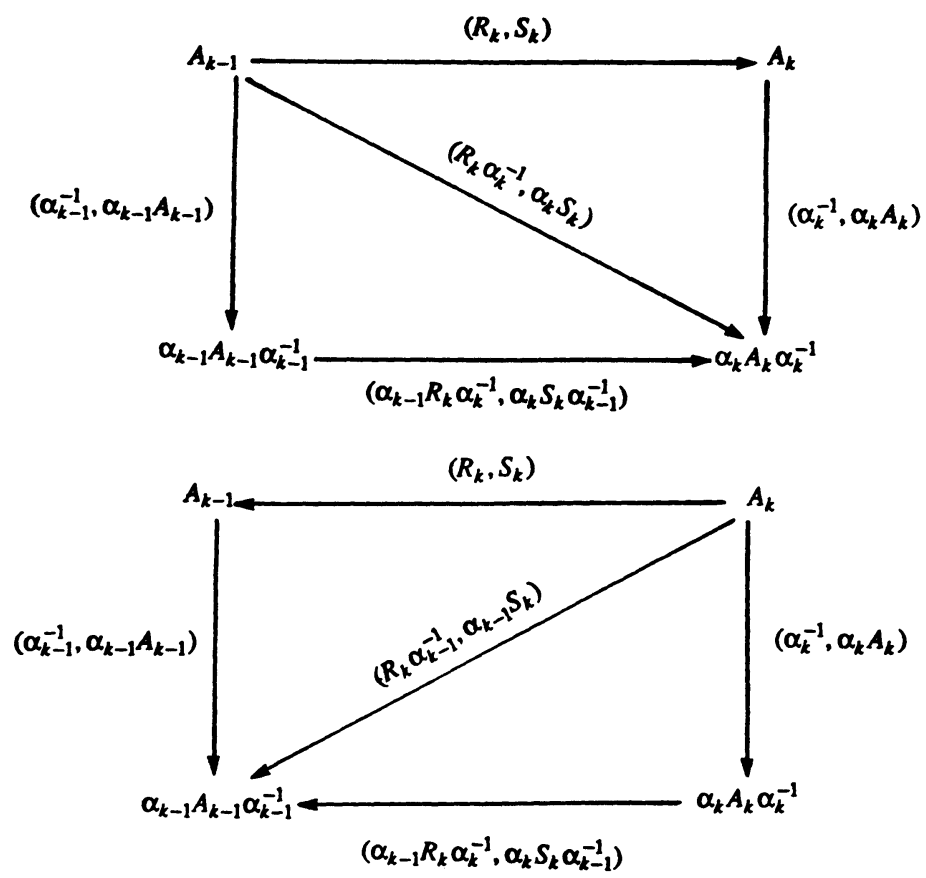

These fit together to provide a homotopy in $\operatorname{RS}(\mathscr{E})$ with end points fixed between the original path $\gamma$ and the path

$$
\gamma\left(\alpha_{0}^{-1}, \alpha_{0} A\right) * \Gamma\left(\alpha_{0}\left(U^{A}\right), U^{B}\right)=\Phi\left(\alpha_{0}\right) .
$$

But $\alpha_{0}=\Theta\left(\gamma^{-1}\right)$. So $\Phi \Theta\left(\gamma^{-1}\right)=\gamma$. This finally completes the proof that $\Phi$ and $\Theta$ are isomorphisms.

REMARK 3.15. The CW-complex $\mathrm{RS}(\mathscr{E})$ is locally compact. This follows from (3.13), the observation in [8] that $P_{A}$ is a locally finite simplicial complex, and the fact that there are only finitely many $(R, S): P \rightarrow P$ for a given square zero-one matrix $P$.

4. $\mathrm{RS}(\mathscr{E})$ is aspherical. We now give the proof of (1.13). Let $\mathrm{RS}(\mathscr{E})_{A}$ denote the component of $\mathrm{RS}(\mathscr{E})$ containing $A$. The universal cover $\widetilde{\mathrm{RS}}_{A}$ of $\mathrm{RS}(\mathscr{E})_{A}$ is the realization of the following simplicial set: The $k$-simplices are pairs $(\gamma, \Delta)$ where

(i) $\Delta$ is a $k$-simplex of $\operatorname{RS}(\mathscr{E})_{A}$ given by the data $\left\langle A_{0}, \ldots, A_{n}\right\rangle$ and $\left(R_{i j}, S_{j i}\right): A_{i} \rightarrow A_{j}$ as in (1.4) and

(ii) $\gamma$ is a homotopy class of paths from the base point $A$ to $A_{0}$. The $i$ th face operator of $\widetilde{R S}$ acts on $\Delta$ just as it does in RS. For $1 \leq i \leq n$, it leaves $\gamma$ unchanged, and for $i=0$ it changes $\gamma$ to 
$\gamma * \gamma\left(R_{1}, S_{1}\right)$. Similarly for the degeneracy operators. The covering map

$$
\widetilde{\mathrm{RS}}(\mathscr{E})_{A} \rightarrow \operatorname{RS}(\mathscr{E})_{A}
$$

is induced by the map of simplicial sets taking $(\gamma, \Delta)$ to $\Delta$.

For each $k$-simplex $(\gamma, \Delta)$, let $\alpha_{0}=\Theta(\gamma)$ and for $1 \leq k \leq n$, let $\alpha_{k}=\Theta\left(\gamma * \gamma\left(R_{0 k}, S_{k 0}\right)\right)$. Let $U_{k}=\alpha_{k}\left(U^{A_{k}}\right) \in P_{A}$. The discussion in $\S 3$ shows $\left\langle U_{0}, \ldots, U_{k}\right\rangle$ is a $k$-simplex in $P_{A}$. Moreover, the correspondence taking $(\gamma, \Delta)$ to $\left\langle U_{0}, \ldots, U_{k}\right\rangle$ is map of simplicial sets. Using (3.14) we obtain a homotopy commutative diagram

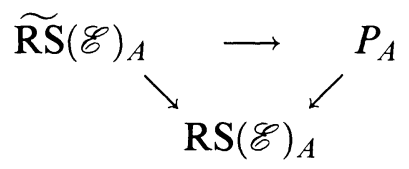

Since $P_{A}$ is contractable [8], we see that

$$
\pi_{n}\left(\widetilde{\mathrm{RS}}(\mathscr{E})_{A}, A\right) \rightarrow \pi_{n}\left(\mathrm{RS}(\mathscr{E})_{A}, A\right)
$$

is the zero homomorphism. On the other hand, it is also an isomorphism for $n \geq 2$ because $\widetilde{\mathrm{RS}}(\mathscr{E})_{A}$ is the universal cover.

\section{REFERENCES}

[1] M. Boyle and W. Krieger, Periodic points and automorphisms of the shift, to appear in Trans. Amer. Math. Soc.

[2] M. Boyle, D. Lind, and D. Rudolph, The automorphism group of a subshift of finite type, preprint, University of Washington/University of Maryland, 1986.

[3] E. G. Effros, Dimensions and $C^{*}$-algebras, CBMS No. 46, Amer. Math. Soc., 1981.

[4] J. Franks, Homology and dynamical systems, CBMS No. 49, Amer. Math. Soc., 1982.

[5] W. Parry and S. Tuncel, Classification Problems in Ergodic Theory, LMS Lecture Notes 67, Cambridge University Press, 1982.

[6] G. Segal, Classifying spaces and spectral sequences, Pub. Math. IHES No. 34, 1968.

[7] E. Spanier, Algebraic Topology, McGraw Hill, 1966.

[8] J. B. Wagoner, Markov partitions and $K_{2}$, Pub. Math. IHES No. 65, 1987, pp. 91-129.

[9] _ Eventual finite generation for the kernel of the dimension group presentation, Trans. Amer. Math. Soc., 317 (1990), 331-350.

Received June 30, 1988 and in revised form September 30, 1988. Partially supported by NSF. 



\section{PACIFIC JOURNAL OF MATHEMATICS EDITORS}

\author{
V. S. VARADARAJAN \\ (Managing Editor) \\ University of California \\ Los Angeles, CA 90024-1555-05 \\ Herbert Clemens \\ University of Utah \\ Salt Lake City, UT 84112 \\ Thomas ENRIGHT \\ University of California, San Diego \\ La Jolla, CA 92093
}

R. FINN

Stanford University

Stanford, CA 94305

Hermann FlaschKa

University of Arizona

Tucson, AZ 85721

VAUGHAN F. R. Jones

University of California

Berkeley, CA 94720

STEVEN KERCKHOFF

Stanford University

Stanford, CA 94305
C. C. MOORE

University of California

Berkeley, CA 94720

Martin SCharlemanN

University of California

Santa Barbara, CA 93106

HAROLd STARK

University of California, San Diego

La Jolla, CA 92093

\section{ASSOCIATE EDITORS}
R. ARENS
E. F. BECKENBACH
B. H. NeumanN
F. WoLF
(1904-1989)
K. YoshidA (1906-1982)

\section{SUP}

UNIVERSITY OF ARIZONA

UNIVERSITY OF BRITISH COLUMBIA

CALIFORNIA INSTITUTE OF TECHNOLOGY

UNIVERSITY OF CALIFORNIA

MONTANA STATE UNIVERSITY

UNIVERSITY OF NEVADA, RENO

NEW MEXICO STATE UNIVERSITY

OREGON STATE UNIVERSITY
UNIVERSITY OF OREGON

UNIVERSITY OF SOUTHERN CALIFORNIA

STANFORD UNIVERSITY

UNIVERSITY OF HAWAII

UNIVERSITY OF TOKYO

UNIVERSITY OF UTAH

WASHINGTON STATE UNIVERSITY

UNIVERSITY OF WASHINGTON 


\section{Pacific Journal of Mathematics}

Vol. 144, No. $1 \quad$ May, 1990

Wojciech Chojnacki, On some totally ergodic functions $\ldots \ldots \ldots \ldots \ldots \ldots 1$

Steven R. Costenoble, Stefan Waner and G. S. Wells, Approximating

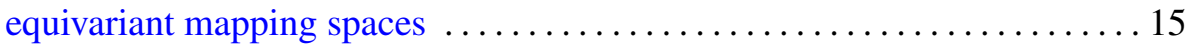

Peter Michael Higgins, A short proof of Isbell's zigzag theorem . . . . . . . 47

Harold H. Johnson, The absolute invariance of conservation laws ........5 51

Edgar Kann, Infinitesimal rigidity of almost-convex oriented polyhedra of arbitrary Euler characteristic $\ldots \ldots \ldots \ldots \ldots \ldots \ldots \ldots \ldots \ldots \ldots \ldots \ldots$

Alan Van Lair, Uniqueness for a nonlinear abstract Cauchy problem . . . . . 105

John B. Little and Kathryn A. Furio, On the distribution of Weierstrass points on irreducible rational nodal curves $\ldots \ldots \ldots \ldots \ldots \ldots \ldots \ldots \ldots 131$

J. S. Okon and Louis Jackson Ratliff, Jr., Reductions of filtrations . . . . . 137

Janusz Pawlikowski, Small subset of the plane which almost contains almost all Borel functions ............................... 155

Sergio A. Tozoni, Vector singular integral operators on a local field ....... 161 John Bason Wagoner, Triangle identities and symmetries of a subshift of

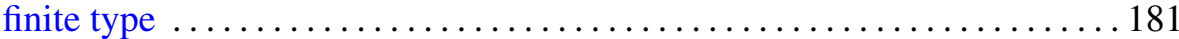

